\title{
Interleukin-6 Blockade as Rescue Therapy in Autoimmune Encephalitis
}

\author{
Russell C. Dale ${ }^{1}$
}

Published online: 16 August 2016

(C) The American Society for Experimental NeuroTherapeutics, Inc. 2016

Autoimmune encephalitis (AE) is an expanding group of inflammatory brain conditions defined by the presence of autoantibodies against cell-surface proteins such as neuronal receptors or synaptic proteins [1]. AE is important to suspect and diagnose, as early immune therapy appears to improve outcomes [2]. With this aim, consensus criteria have been recently proposed to diagnose $\mathrm{AE}$ [1]. To date, therapeutic approaches have borrowed from the treatment of rheumatologic and other immune-mediated central nervous system (CNS) diseases. First-line therapies typically include corticosteroid plus intravenous immunoglobulin and/or plasma exchange. If patients do not respond to first-line therapy, secondline therapy with rituximab (a monoclonal antibody targeting $\mathrm{CD} 20^{+} \mathrm{B}$ cells) and/or cyclophosphamide should be considered [3]. Despite this therapeutic pathway, a significant proportion of patients will not respond and continue to have neurologic and cognitive impairments, which can be permanent. Given the rarity of AE, therapeutic understanding has mostly derived from retrospective uncontrolled cohort studies, using modified Rankin scores (mRS) as outcome measures, which define functional impairments using a simple $0-6$ scoring system [3]. The outcomes of patients are influenced by the timing of therapy, and earlier diagnosis and treatment generally improve outcomes $[2,4]$. A further confounder is that patients

This comment refers to the article available at: http://dx.doi.org/10.1007 /s13311-016-0442-6.

Russell C. Dale

russell.dale@health.nsw.gov.au

1 Brain Autoimmunity Group, Institute for Neuroscience and Muscle Research, the Children's Hospital at Westmead, University of Sydney, Sydney, Australia with anti- $N$-methyl-D-aspartate receptor encephalitis, the most common $\mathrm{AE}$, continue to improve for 2 years, and so short outcome studies will not accurately capture the final outcomes [3].

When second-line therapy fails or persistent neurologic problems remain (which occurs in $\sim 20-40 \%$ of patients), the therapeutic options are less clear, and there is a lack of guidance. Options could include further broad-spectrum immune suppression such as intrathecal methotrexate [5], or more targeted immune therapy such as monoclonal antibodies against cytokines/chemokines or cellular targets [6].

The study in this issue of Neurotherapeutics by Lee et al. [7] describes the use of the monoclonal antibody tocilizumab [an interleukin (IL)-6 blocker] in patients with $\mathrm{AE}$ who have failed to respond to rituximab and continue to have significant impairments. In this retrospective institutional cohort study, carried out over a 3 -year period, of 185 patients with suspected and definite AE, 91 patients had received first-line therapy and rituximab yet had persistent neurologic deficits 1 month after rituximab. The cohort of 91 patients with $\mathrm{AE}$ who were unresponsive to rituximab was split into 3 groups: patients who received tocilizumab $(n=30)$, those who were redosed with rituximab $(n=31)$, and those who received no further immune therapy (observational group, $n=30$ ). Despite the uncontrolled retrospective design, the patients at study entry were similar in clinical characteristics, duration of disease, and disease severity (using $\mathrm{mRS}$ ). A favorable response was defined as a reduction of $\mathrm{mRS}$ by $\geq 2 \mathrm{mRS}$ points, or a $\mathrm{mRS}$ score $\leq 2$ at follow-up. The patients in the tocilizumab group did significantly better than the patients in both the rituximab re-dosing group and observational group, at 1 month, 2 months, and final follow-up [7]. The safety profile of tocilizumab appeared reasonable, with no reported infusion reactions, although $3 / 30$ had measurable neutropenia $(<1000 / \mathrm{dl})$ without described complications. 
There are a number of limitations to the study. First, although the cohort fulfilled criteria for "possible AE" [1], only $31 / 91$ patients in the cohort had defined autoantibodies, therefore, "definite" AE, and the majority of the patients had "seronegative AE" (the cohort was therefore heterogenous). Although this is a relative weakness of the study, this is a "real-life" issue as current testing leaves a diagnosis of suspected but seronegative AE in a significant proportion of cases. A further weakness of the study is the relative lack of a rationale for the use of IL- 6 blockade in the patients described in this report, as this cohort did not have IL-6 measured in serum or cerebrospinal fluid. IL-6 is a cytokine with pleiotropic effects and has a major role in T- and B-cell inflammatory cascades. Consequently, IL-6 is elevated in a very broad spectrum of CNS inflammation, particularly autoimmune CNS diseases [8]. Tocilizumab has been used predominantly in rheumatoid arthritis to induce and maintain remission from disease. Recently, tocilizumab has been used to treat neuromyelitis optica (NMO) [9], an autoimmune CNS disease with pathogenic antiaquaporin-4 autoantibodies, although the immunopathogenesis of NMO is complex with pathological evidence of autoantibody, complement, T cells, B cells, neutrophils, and eosinophils [10]. IL-6 is elevated in NMO attacks, is involved in autoantibody production, and is used as a disease biomarker [11]. By contrast, the literature regarding IL-6 in AE is small and only now emerging, although some small studies have shown that IL-6 is elevated in $\operatorname{AE}[12,13]$. Other cytokines/chemokines such as $\mathrm{C}$-X-C motif chemokine ligand 13 have also been shown to be elevated, and sustained elevation of CSF C-X-C motif chemokine ligand 13 correlates with disease severity and failure to improve [14].

Despite these limitations, this study by Lee et al. [7] is important and provocative, and based on these data, IL-6 blockade should be considered in patients with $\mathrm{AE}$ who fail to respond to rituximab or other second-line therapies.

However, there are a number of questions about tocilizumab and its role in the treatment of AE. First, does tocilizumab act peripherally only, or centrally within the CNS? Can tocilizumab cross the blood-brain barrier, and is this important to the potential effectiveness of tocilizumab? Tocilizumab has been predominantly used in rheumatoid arthritis to maintain remission, and the speed of action of IL-6 blockade has not been well studied in CNS disease. For an agent to be a useful acute rescue therapy in $\mathrm{AE}$, the speed of immune suppression needs to be rapid; reassuringly, the data by Lee et al. [7] appear to show clinical benefit within a month of commencing tocilizumab therapy.

This study also provokes a discussion regarding the issue of "broad-spectrum" versus "targeted" immune therapy. Therapies that target a specific molecule will only work if that molecule is central to the disease process. In complex acquired autoimmune diseases, although there are often specific initiators of disease, in established disease one might expect more complex and diverse involvement of the immune system with a mixture of "primary" and "secondary" immune activation. Therefore, targeted therapies, although appealing, may not always work. Targeting IL6 is relatively appealing in autoimmune CNS disease given its established role in CNS inflammation and its pleiotropic stimulatory role [8].

In summary, the study by Lee et al. [7] provides a good option to clinicians faced with a patient with AE or suspected $\mathrm{AE}$ who is not responding to conventional therapy. Second, the study emphasizes that if a patient is not improving, it is important to consider using an agent with a different mechanism of action, rather than re-dosing a drug that appears to be ineffective. One of the most challenging issues for the treating clinician is defining the time point of treatment failure, and when to escalate treatment; Lee et al. [7] defined treatment failure as an "inadequate clinical response" with "lack of obvious improvement of the most disabling symptoms, based on the clinician's opinion" 1 month after completion of rituximab [7]. Although this definition is subjective, it is a "real-life" definition at the bedside. The use of 1 month as a time point seems reasonable, as delay is generally considered to result in worsening outcomes. Defining treatment failure and the need to escalate treatment remains one of the most challenging issues in the treatment of $\mathrm{AE}$ and should be the focus of future research.

Required Author Forms Disclosure forms provided by the authors are available with the online version of this article.

\section{References}

1. Graus F, Titulaer MJ, Balu R, et al. A clinical approach to diagnosis of autoimmune encephalitis. Lancet Neurol 2016;15:391-404.

2. Nosadini M, Mohammad SS, Ramanathan S, Brilot F, Dale RC. Immune therapy in autoimmune encephalitis: a systematic review. Expert Rev Neurother 2015;15:1391-419.

3. Titulaer MJ, McCracken L, Gabilondo I, et al. Treatment and prognostic factors for long-term outcome in patients with anti-NMDA receptor encephalitis: an observational cohort study. Lancet Neurol 2013;12:157-65.

4. Byrne S, Walsh C, Hacohen Y, et al. Earlier treatment of NMDAR antibody encephalitis in children results in a better outcome. Neurol Neuroimmunol Neuroinflamm 2015;2:e130.

5. Tatencloux S, Chretien P, Rogemond V, Honnorat J, Tardieu $\mathrm{M}$, Deiva K. Intrathecal treatment of anti-N-Methyl-Daspartate receptor encephalitis in children. Dev Med Child Neurol 2015;57:95-9.

6. Dale RC, Brilot F, Duffy LV, et al. Utility and safety of rituximab in pediatric autoimmune and inflammatory CNS disease. Neurology 2014;83:142-50.

7. Lee WJ, Lee ST, Moon J, et al. Tocilizumab in Autoimmune Encephalitis Refractory to Rituximab: An Institutional Cohort Study. Neurotherapeutics 2016. doi:10.1007/s13311-016-0442-6.

8. Kothur K, Wienholt L, Brilot F, Dale RC. CSF cytokines/ chemokines as biomarkers in neuroinflammatory CNS disorders: A systematic review. Cytokine 2016;77:227-37. 
9. Araki M, Matsuoka T, Miyamoto K, et al. Efficacy of the anti-IL-6 receptor antibody tocilizumab in neuromyelitis optica: a pilot study. Neurology 2014;82:1302-6.

10. Lucchinetti CF, Guo Y, Popescu BF, Fujihara K, Itoyama Y, Misu T. The pathology of an autoimmune astrocytopathy: lessons learned from neuromyelitis optica. Brain Pathol 2014;24:83-97.

11. Chihara N, Aranami T, Sato W, et al. Interleukin 6 signaling promotes anti-aquaporin 4 autoantibody production from plasmablasts in neuromyelitis optica. Proc Natl Acad Sci U S A 2011;108:3701-6.
12. Byun JI, Lee ST, Moon J, et al. Distinct intrathecal interleukin-17/ interleukin-6 activation in anti-N-methyl-d-aspartate receptor encephalitis. J Neuroimmunol 2016;297:141-7.

13. Ygberg S, Fowler A, Wickstrom R. Cytokine and Chemokine Expression in CSF May Differentiate Viral and Autoimmune NMDAR Encephalitis in Children. J Child Neurol 2016.

14. Leypoldt F, Hoftberger R, Titulaer MJ, et al. Investigations on CXCL13 in anti-N-methyl-D-aspartate receptor encephalitis: a potential biomarker of treatment response. JAMA Neurol 2015;72: 180-6. 